\title{
Thalidomide treatment of mucosal ulcerations in HIV infection
}

\author{
R A Solèr, C Migliorati, H van Waes, D Nadal
}

\begin{abstract}
Pain relief and resolution of oral and perianal ulceration after treatment with thalidomide in a 14 year old girl with vertically acquired HIV infection is reported. (Arch Dis Child 1996; 74: 64-65)
\end{abstract}

Keywords: thalidomide, HIV.

Recurrent mucosal ulcers of unknown aetiology are frequent in adults with advanced HIV infection, may contribute substantially to morbidity, and are often refractory to various treatments. ${ }^{1}$ We report a child infected with HIV whose chronic mucosal ulcers resolved on treatment with thalidomide.

Infectious Diseases

Unit, University

Children's Hospital of

Zurich,

Steinwiesstrasse 75 ,

CH-8032 Zurich,

Switzerland

R A Solèr

D Nadal

Public Oral Health

Clinic, Zurich

C Migliorati

Outpatient Dental

Clinic, Dental

Institute, University of

Zurich

$\mathrm{H}$ van Waes

Correspondence to: Dr Nadal.

Accepted 24 July 1995

\section{Case report \\ A 14 year old African girl with vertically acquired HIV infection developed five painful ulcers (diameter 2 to $12 \mathrm{~mm}$ ) on the tongue and gingiva (figure A). She had been well until the age of 9 years when she experienced her first of several episodes of pneumonia. In the following years she suffered from recurrent oral candidiasis, lymphocytic interstitial pneu- monitis, and one episode of Pneumocystis carinii pneumonia. Since the age of 12 years, the CD4+ cell count was known to be $0 / \mu l$. Zidovudine (azidothymidine) started at the age of 11 years had to be discontinued because of neutropenia. She was then treated with didanosine.}
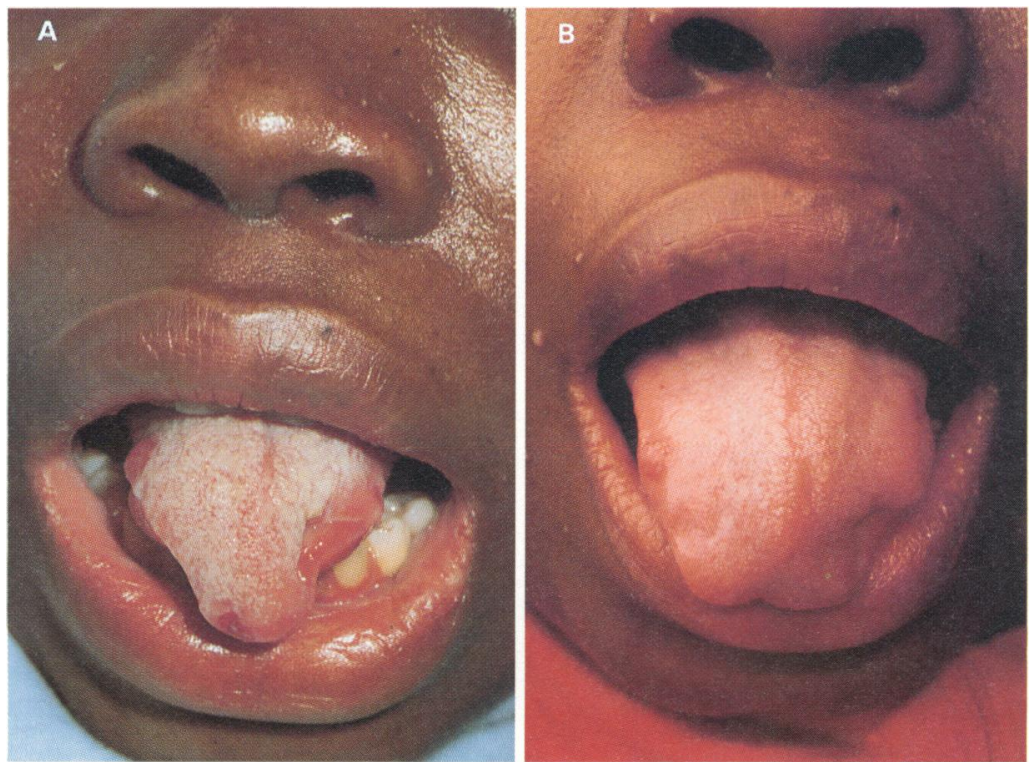

Response of oral ulcers to thalidomide in an HIV infected 14 year old girl. (A) Before treatment and $(B)$ after three weeks of treatment.
Cultures from the oral lesions were negative for bacteria, mycobacteria, fungi, and viruses as was electron microscopy for herpes particles. Treatment with anaesthetic and disinfectant mouth washes, topical corticosteroids, and oral fluconazole $(100 \mathrm{mg} /$ day $)$ gave only slight relief. A painful haemorrhoidal node with several ulcerations developed. Antihaemorrhoidal suppositories and ointments were without success. At haemorrhoidectomy the deepest oral lesion was biopsied. Conventional histology including haematoxylin-eosin, Ziehl-Neelsen, Gram, Giemsa, and Grocott stains revealed chronic inflammation, but failed to detect any infectious agent. In situ hybridisation and immune histochemical analysis for herpes viruses were negative.

Prednisone $2 \mathrm{mg} / \mathrm{kg} /$ day for four days produced some pain relief and healing of the mouth ulcers. However, it was discontinued because of suspected acute infection. She then deteriorated.

Oral thalidomide $(100 \mathrm{mg} /$ day (3 $\mathrm{mg} / \mathrm{kg} /$ day); kindly provided by Grünenthal, Germany) resulted in dramatic pain relief within three days, complete resolution of ulcers within three weeks (figure B), and in substantial improvement of the chronic gingivitis. Rectal pain disappeared and the haemorrhoidal node almost vanished. Apart from moderate fatigue, no adverse effects were observed.

\section{Discussion}

Dramatic responses of oropharyngeal, oesophageal, and rectal ulcerations to thalidomide, $200 \mathrm{mg}$ for 14 days, have been recently reported in 19 of $20 \mathrm{HIV}$ infected adults. ${ }^{1}$ The mechanism of action is not known. In vitro studies have suggested that thalidomide inhibits the production of tumour necrosis factor- $\alpha$ by activated monocytes, ${ }^{2}$ induces $T$ helper cell type 2 , and inhibits $T$ helper cell type 1 cytokine production. ${ }^{3}$ The present report demonstrates that HIV infected children with mucosal ulcers of unknown aetiology may benefit from thalidomide treatment.

Recently, thalidomide has been employed successfully in the treatment of graft-versushost disease after bone marrow transplantation, systemic lupus erythematosus, and Behçet's disease, and for the prevention of cardiac allograft rejection, suggesting that the drug is immunosuppressive. ${ }^{1}$

In the 1950s thalidomide was used as a sedative and antiemetic in pregnant women. Teratogenicity resulted in its withdrawal from use. ${ }^{4}$ Thalidomide for our patient was supplied by the manufacturer only under stringent 
conditions requiring individual patient use protocol, after informed consent by the patient and parents.

Because chronic mucosal ulceration in HIV infected patients may be extremely painful, debilitating and refractory to treatment, ${ }^{5}$ thalidomide should be considered provided the teratogenic effects are disclosed and it is certain the patient is not at risk for pregnancy.

1 Paterson DL, Georghiou PR, Allworth AM, Kemp RJ. Thalidomide as treatment of refractory aphthous ulceration related to human immunodeficiency virus infection. Clin Infect Dis 1995; 20: 250-4.

2 Moreira AL, Sampaio EP, Zmuidzinas A, Frindt P, Smith KA, Kaplan G. Thalidomide exerts its inhibitory action on tumor necrosis factor $\alpha$ by enhancing mRNA degradation. f Exp Med 1993; 177: 1675-80. 3 McHugn SM, Rifkin IR, Deighton J, Wilson AB, Lachman PJ, Lockwood CM. The immunosuppressive drug thalidomide induces T helper cell type 2 (Th2) and concomitantly mide induces Thelper cell type 2 ( 12 ) and concomitantly inhibits Th1 cytokine production in mitogen- and antigenstimulated human peripheral blood mon

cultures. Clin Exp Immunol 1995; 99: 160-7.
McBride WG. Thalidomide and congenital abnormalities McBride WG. Thalidomid
Lancet 1961; ii: 1358.

5 MacPhail LA, Greenspan D, Greenspan JS. Recurrent aphthous ulcers in association with HIV infection. Diagnosis and treatment. Oral Surg Oral Med Oral Patho 1992; 73: 283-8. 